\title{
THE UNIMODALITY CONJECTURE FOR CONVEX POLYTOPES
}

\author{
BY ANDERS BJÖRNER
}

A sequence of integers $a_{0}, a_{1}, \ldots, a_{n}$ is said to be unimodal if

$$
a_{0} \leqslant a_{1} \leqslant \cdots \leqslant a_{k} \geqslant a_{k+1} \geqslant \cdots \geqslant a_{n}
$$

for some $k, 0 \leqslant k \leqslant n$. Empirical evidence has suggested that some important numerical sequences in combinatorics are unimodal. For instance, unimodality conjectures have been made for the coefficients of the chromatic polynomial of a graph, for the independence numbers of a matroid and for the face numbers of a convex polytope. The purpose of the present note is to announce that the unimodality conjecture for convex polytopes is false: there are 24-dimensional counterexamples on around a trillion vertices. It appears that the unimodality conjecture for convex polytopes was first suggested by T. S. Motzkin in the late 1950's, and was communicated by him at the convexity symposium in Seattle in 1961 [3]. The unimodality question for polytopes was later independently raised by $D$. Welsh [4]. The present result suggests that the unimodality conjectures which have been made for shellable structures, including the initially cited examples, should be approached with caution: counterexamples may be hard to find only because of their size.

For the basic definitions in polyhedral theory we refer to Grünbaum's book [2], to which we adhere in matters of terminology and notation.

THEOREM 1. Suppose that a triangulation of the $(d-1)$-sphere has $f_{i}$ $i$-dimensional faces, $0 \leqslant i \leqslant d-1, d \geqslant 3$. Then

$f_{0}<f_{1}<\cdots<f_{[d / 2]-1} \leqslant f_{[d / 2]}$ and $f_{[3(d-1) / 4]}>\cdots>f_{d-2}>f_{d-1}$.

The proof is based on the Dehn-Sommerville equations [2, p. 146], so the result is true also for certain spaces more general than spheres. Complementing the inequalities from Theorem 1 with some case-by-case checking, we can show that the $f$-vectors $\left(f_{0}, f_{1}, \ldots, f_{d-1}\right)$ of simplicial $d$-polytopes are unimodal for $d \leqslant 15$. However, the unimodality conjecture is not true even for the class of simplicial polytopes.

Received by the editors May 30, 1980; presented to the Society, April 25, 1980 at Davis, California.

1980 Mathematics Subject Classification. Primary 52A25; Secondary 05A20. 
THEOREM 2. There is a 24-dimensional simplicial polytope on $2.6 \times 10^{11}$ vertices for which $f_{14}>f_{15}<f_{16}$. Hence, $f$-vectors of simplicial convex polytopes need not be unimodal.

To prove this result we employ the Billera-Lee-McMullen condition [1] for a vector to be the $f$-vector of a simplicial polytope. This condition involves numerical invariants of certain systems of monomials, and by counting such invariants ("0-sequences") we get the following lower bound for the number $f_{s}(v, d)$ of $f$ vectors of simplicial $d$-polytopes on $v$ vertices.

ThEOREM 3. For $(p,[d / 2]) \neq(1,3)$

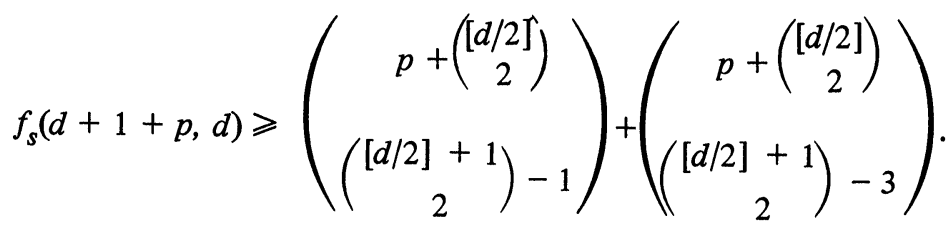

It turns out that this lower bound is asymptotically sharp in the sense that $f_{s}(v, d)$ has polynomial growth in $\left.v: f_{s}(v, d) \sim v^{\cdot([d / 2]+1}\right)-1, d$ fixed.

Note added in proof. Since this paper was written C. W. Lee and the author have independently found 20-dimensional simplicial polytopes on around $4.2 \times 10^{12}$ vertices for which $f_{11}>f_{12}<f_{13}$.

\section{REFERENCES}

1. L. J. Billera and C. W. Lee, Sufficiency of McMullen's conditions for f-vectors of simplicial polytopes, Bull. Amer. Math. Soc. (N.S.) 2 (1980), $181-185$.

2. B. Grünbaum, Convex polytopes, Wiley, New York and London, 1967.

3. B. Grünbaum and V. Klee, personal communication.

4. D. J. A. Welsh, Problem 1, Combinatorics (D. J. A. Welsh and D. R. Woodall, (eds.)), Institute of Math. and Appl., Oxford, 1972, p. 357.

DEPARTMENT OF MATHEMATICS, UNIVERSITY OF CALIFORNIA, SAN DIEGO, LA JOLLA, CALIFORNIA 92093

Current address: Department of Mathematics, University of Stockholm, Box 6701, 11385 Stockholm, Sweden 